\title{
Direct and indirect costs and cost-driving factors in adults with tuberous sclerosis complex: a multicenter cohort study and a review of the literature
}

Johann Philipp Zöllner ${ }^{1,2}$, Janina Grau 1,2, Felix Rosenow ${ }^{1,2}$, Matthias Sauter ${ }^{3}$, Markus Knuf ${ }^{4,5}$, Gerhard Kurlemann ${ }^{6}$, Thomas Mayer ${ }^{7}$, Christoph Hertzberg ${ }^{8}$, Astrid Bertsche ${ }^{9}$, Ilka Immisch ${ }^{10}$, Karl Martin Klein 1,2,11, Susanne Knake ${ }^{10}$, Klaus Marquard ${ }^{12}$, Sascha Meyer ${ }^{13}$, Anna H. Noda ',2, Felix von Podewils ${ }^{14}$, Hannah Schäfer ${ }^{15,16}$, Charlotte Thiels ${ }^{17}$, Laurent M. Willems ${ }^{1,2}$, Bianca Zukunft ${ }^{18}$, Susanne Schubert-Bast ${ }^{1,2,19}$ and Adam Strzelczyk ${ }^{1,2,10^{*}}$ (D)

\begin{abstract}
Background: Tuberous sclerosis complex (TSC) is a monogenetic, multisystem disorder characterized by benign growths due to TSC1 or TSC2 mutations. This German multicenter study estimated the costs and related cost drivers associated with organ manifestations in adults with TSC.
\end{abstract}

Methods: A validated, three-month, retrospective questionnaire assessed the sociodemographic and clinical characteristics, organ manifestations, direct, indirect, out-of-pocket (OOP), and nursing care-level costs among adult individuals with TSC throughout Germany from a societal perspective (costing year: 2019).

Results: We enrolled 192 adults with TSC (mean age: $33.4 \pm 12.7$ years; range: $18-78$ years, $51.6 \%$ [ $n=99$ ] women). Reported TSC disease manifestations included skin (94.8\%) and kidney and urinary tract (74\%) disorders, epilepsy (72.9\%), structural brain defects (67.2\%), psychiatric disorders (50.5\%), heart and circulatory system disorders (50.5\%), and lymphangioleiomyomatosis (11.5\%). TSC1 and TSC2 mutations were reported in 16.7\% and 25\% of respondents, respectively. Mean direct health care costs totaled EUR 6452 (median EUR 1920; 95\% confidence interval [CI] EUR 5533-7422) per patient over three months. Medication costs represented the major direct cost category (77\% of total direct costs; mean EUR 4953), and mechanistic target of rapamycin (mTOR) inhibitors represented the largest share (68\%, EUR 4358). Mean antiseizure drug (ASD) costs were only EUR 415 (6\%). Inpatient costs (8\%, EUR 518) and outpatient treatment costs (7\%; EUR 467) were important further direct cost components. The mean care grade allowance as an approximator of informal nursing care costs was EUR 929 (median EUR 0; 95\% CI EUR 780-1083) over three months. Mean indirect costs totaled EUR 3174 (median EUR 0; 95\% CI EUR 2503-3840) among working-age individuals ( $<67$ years in Germany). Multiple regression analyses revealed mTOR inhibitor use and persistent seizures as independent cost-driving factors for total direct costs. Older age and disability were independent cost-driving factors

\footnotetext{
*Correspondence: strzelczyk@med.uni-frankfurt.de

1 Epilepsy Center Frankfurt Rhine-Main, Department of Neurology,

Goethe-University Frankfurt, Schleusenweg 2-16, 60528 Frankfurt am Main, Germany

Full list of author information is available at the end of the article
}

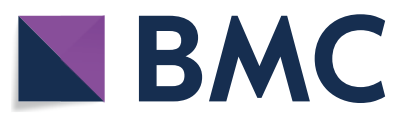

(c) The Author(s) 2021. Open Access This article is licensed under a Creative Commons Attribution 4.0 International License, which permits use, sharing, adaptation, distribution and reproduction in any medium or format, as long as you give appropriate credit to the original author(s) and the source, provide a link to the Creative Commons licence, and indicate if changes were made. The images or other third party material in this article are included in the article's Creative Commons licence, unless indicated otherwise in a credit line to the material. If material is not included in the article's Creative Commons licence and your intended use is not permitted by statutory regulation or exceeds the permitted use, you will need to obtain permission directly from the copyright holder. To view a copy of this licence, visit http://creativecommons.org/licenses/by/4.0/. The Creative Commons Public Domain Dedication waiver (http://creativeco mmons.org/publicdomain/zero/1.0/) applies to the data made available in this article, unless otherwise stated in a credit line to the data. 
for total indirect costs, whereas epilepsy, psychiatric disease, and disability were independent cost-driving factors for nursing care costs.

Conclusions: This three-month study revealed substantial direct healthcare, indirect healthcare, and medication costs associated with TSC in Germany. This study highlights the spectrum of organ manifestations and their associated treatment needs in the German healthcare setting. Trial registration: DRKS, DRKS00016045. Registered 01 March 2019, http://www.drks.de/DRKS00016045.

Keywords: TSC, Angiomyolipoma, Seizure, Epilepsy, Subependymal giant cell astrocytoma, Costs, Sociodemographic characteristics, Genetics, Anticonvulsant, MTOR inhibitor

\section{Key points}

- This comprehensive study measured the direct and indirect costs of individuals with TSC and their caregivers

- Mean total direct costs (healthcare and non-healthcare) were estimated at EUR 6452 over three months

- Medication, particularly mTOR inhibitors, were major direct cost components, followed by hospitalization and outpatient treatment

- Mean total indirect costs were estimated at EUR 3174 over three months; with an inability to work being the largest factor

- Total cost is driven by the number of TSC manifestations and affected organ systems

\section{Background}

Tuberous sclerosis complex (TSC) is a rare multisystem, monogenetic disorder. The estimated incidence rate of definite or possible TSC in Germany is approximately $1: 6760$ to $1: 13,520$ live births [1]. The prevalence of TSC was generally underestimated until recently due to incomplete penetrance and the existence of considerable interindividual phenotypic variability among those affected by TSC [1-6]. In TSC, benign tumors manifest in multiple organ systems, and the clinical manifestations of TSC can vary throughout life, with tumors presenting in most organs, especially the skin, brain, and kidneys. Most individuals with TSC suffer from structural epilepsy due to the formation of cortical tubers or other cortical malformations [7]. The clinical picture for each individual may differ considerably and can range from very limited manifestations to severe impairments that require nursing assistance $[4,7]$. Individuals are commonly diagnosed with TSC in response to the development of epileptic seizures, particularly the development of epileptic spasms at a young age, often within the first six months after birth [8]. Other common first findings include skin manifestations, and TSC can sometimes be suspected even before birth due to cardiac rhabdomyoma [7]. Neuropsychiatric problems, including intellectual disability, autism, sleep difficulties, and aggression are frequent in children with TSC, they have been associated with early seizure onset, epileptic spasms, and TSC2 gene mutations, among other factors [7]. During adolescence, renal manifestations, such as angiomyolipoma (AML) and subependymal giant cell astrocytoma (SEGA) can become burdensome [9]. Renal AML tends to grow during adulthood, necessitating life-long surveillance [10]. Pulmonary manifestations such as lymphangioleiomyomatosis (LAM) almost exclusively affect adult women with TSC [11].

TSC is caused by a loss-of-function mutation in one of two tumor suppressor genes, TSC1 and TSC2 (ratio 1:3.4, as reported in [12]), which is inherited in an autosomaldominant fashion. However, the majority of cases appear to occur due to de novo pathogenic variants. Genetic mosaicism and deep intronic mutations may also be causative, particularly among the $15 \%$ of cases for which definitive hereditary pathogenic variants cannot be identified, despite a definite clinical diagnosis of TSC [12]. A loss-of-function mutation in either TSC1 or TSC2 leads to the overactivation of the mechanistic target of rapamycin (mTOR) pathway, which results in changes in cell growth, the promotion of cell proliferation, and the disruption of cellular energy homeostasis, ultimately promoting tumorigenesis [13]. Treatment with mTOR inhibitors can address this downstream deregulation as they prevent epileptogenesis and possibly the development of other organ manifestations [14].

The burden of illness associated with TSC is considerable and can vary according to the complex and multifaceted disease manifestations [15-17]. Several studies published during the last two decades have examined the cost-of-illness (COI) and COI predictors in TSC. However, only a few have addressed both direct costs and related cost drivers, and no study has examined the indirect costs incurred by adult individuals with TSC. Furthermore, the majority of these studies evaluated individuals with TSC before the availability of mTOR inhibitors, such as everolimus, which are now used to treat various organ manifestations associated with TSC $[4,18]$. 
Thus, the present study aimed to provide a comprehensive analysis of the direct and indirect costs and potential cost-driving factors associated with TSC by surveying a large, multicenter cohort of adults with TSC in Germany.

\section{Methods}

\section{Patients and recruitment}

The present study was designed as a cross-sectional, multicenter survey that enrolled individuals with TSC throughout Germany (Berlin, Bochum, Dresden [Radeberg], Frankfurt, Greifswald, Homburg, Kempten, Marburg, München, Münster [Lingen], Rostock, Stuttgart, and Wiesbaden) and through the German TSC patient advocacy group (Tuberöse Sklerose Deutschland e.V., Wiesbaden, Germany).

\section{Survey methods}

After receiving written informed consent from the patients or their legal guardians (if applicable), all individuals with TSC were deemed eligible for study inclusion. We based the diagnostic criteria for TSC on the latest recommendations established by the 2012 international TSC consensus conference [19]. We identified seven primary manifestation categories affected by TSC, including epilepsy, structural brain defects, psychiatric, heart/circulatory system disorders, kidney and urinary tract disorders, dermatological system manifestations, respiratory system manifestations, and other manifestations [16]. The seizure and epilepsy syndrome classifications were adapted to the latest definitions established by the International League against Epilepsy (ILAE) [20, 21]. This study received ethical approval and was registered with the German Clinical Trials Register (DRKS00016045; Universal Trial Number: U11111229-4714). We closely followed the STROBE guidelines (Strengthening The Reporting of Observational Studies in Epidemiology) [22].

We asked individuals with TSC to complete a retrospective questionnaire based on their experiences during the previous three months. The questionnaire was validated in earlier studies [23-26] and we adapted it for use in individuals with TSC. The questionnaire included 36 questions relating to disease characteristics (e.g., genetics, affected organ systems, seizures, medications, and additional symptoms), healthcare resource use (e.g., healthcare visits, accidents, and emergency care), and social conditions. Paper questionnaires in German were sent to individuals with TSC between February and July 2019.

\section{Costing methods}

The aim of this study was to calculate the specific genuine costs associated with TSC, rather than those associated with conditions unrelated to TSC. Therefore, we asked individuals in detail whether the medications, services, and other resources that were consumed were associated with particular organ manifestations of TSC. We evaluated costs using a bottom-up approach from the perspective of the statutory health insurer ("Gesetzliche Krankenversicherung" [GKV]) and society as a whole. The cost categories that were included in the analysis were direct health service costs, patients' out-of-pocket (OOP) expenses, care grade allowances as approximation of informal care costs, and indirect costs. We evaluated these costs according to the German recommendations for performing health economic evaluations [27].

\section{Direct health care costs}

We obtained information regarding the direct health service costs from the literature and from standard reference sources for Germany, which were estimated as previously described [23, 26]. Direct health costs included specifically inpatient stays, outpatient visits, medicines (antiseizure drugs [ASDs], mTOR inhibitors, other prescription drugs, over-the-counter drugs, and emergency medications), medical aids, healthcare professional visits, emergency transportation, diagnostic studies, specific diets, individuals' copayments, rehabilitation costs, private transport costs and copayments for therapies. We based drug costs on the Drug Prescription Report of 2019 ("Arzneiverordnungs-Report") [28], which is an index of available medicines and their average prices in Germany. We standardized the costs of inpatient and outpatient care, specialist care, therapies, and diagnostic studies according to the method described by Bock et al. [29] and physician fee scales (Einheitlicher Bewertungsmaßstab) [30]. Costs were inflated to 2019 levels using the consumer price index for Germany and were expressed in both annual and 3-month terms in 2019 Euro.

\section{Out-of-pocket expenses}

All OOP expenses (copayments) that were reported were considered to be accounted for when supply-side cost estimates were calculated based on resource utilization (ancillary treatments, medical aids, healthcare professionals, and emergency transportation), and these OOP expenses were therefore not included in the calculation of total direct costs. We reported OOP expenses explicitly and added them to the total direct healthcare costs when supply-side utilization estimates were not available (care and supervision, healing agents, and diets) or when expenditures existed beyond the formal healthcare setting (alternative and occupational therapies and equipment costs). 


\section{Care grade allowances as approximation of informal care costs}

We calculated the average care grade allowances [31] under the assumption that nursing services were provided by family members. Care grade allowances are the basis on which the German statutory care insurance pays care allowances. Care grade allowances are determined by the grade of necessary patient care, distinguished by levels $1-5$ on the "Pflegegrade" scale. We used care grade costs as an approximation of informal care costs, and we separately reported any additional care costs reported by the respondents. While care grade allowances do not fully reflect the extent of informal care costs, we used them as a compromise between the goal of capturing a large set of individuals and the feasibility of assessing extent of informal care on an individual level.

\section{Indirect costs}

We calculated productivity losses due to TSC (days off, inability to work, reductions in working hours, or early retirement) using the human capital approach for patients of working age (i.e., below the age of 67). The mean annual gross wage of EUR 44,964 in 2019 [32] was used to calculate the productivity costs for each patient. For days taken off work, gross wages were calculated as EUR 215.14 per calendar day, and daily income was multiplied by total days off [24].

\section{Grouping of questionnaire items}

We collated some questionnaire items into groups when presenting the results. Specifically, the term "ancillary costs" includes physiotherapy, speech therapy, occupational therapy, acupuncture, hippotherapy and other ancillary costs. The term "healthcare professionals" includes neurologists, general practitioners (GPs), orthopedic surgeons, child psychiatrists, alternative medicine practitioners, homeopathy practitioners, dietitians and other specialists. The term "diagnostic studies" includes electroencephalography (EEG), blood tests, magnetic resonance imaging (MRI) or computed tomography (CT) scans, X-rays and other diagnostic studies.

For a detailed overview of the costing sources used, please refer to the Addtional file 1: Supplementary material.

\section{Statistical analysis}

We conducted statistical analysis using IBM SPSS Statistics, version 26 (IBM Corp., Armonk, NY, USA). We summarized the variables of interest using the mean, median, and standard deviation (SD). For cost data, we calculated $95 \%$ confidence intervals (CI), using the bootstrapcorrected and accelerated (BCa) method with $n=2000$ repetitions to estimate parameters robust to skewed distributions and outliers [33, 34]. Due to the small population of TSC and related statistical challenges [35, 36], we refrained from a power calculation or a predefined number of participants, and aimed to include all potential patients with TSC in Germany. We compared groups using adequate parametric and nonparametric tests after testing for the normality of distribution. The significance level was assumed at $p<0.05$. We investigated the relationships between an individual's clinical characteristics and TSC-related costs using multivariate linear regression using the $\mathrm{BCa}$ method with 2000 repetitions. Total direct, total indirect, and nursing care-level costs were regressed against a set of clinical variables, which we selected following univariate analysis and according to evidence presented by previous cost-of-illness studies examining TSC [16, 37, 38]. We tested all variables for interactions and collinearity. To identify independent predictors of costs, we performed standard multiple linear regression analysis using the bootstrapping technique and applied a Bonferroni correction for multiple testing.

\section{Results}

\section{Demographic and clinical characteristics}

One hundred and ninety-two adults with TSC completed the questionnaire. The mean participant age was 33.4 years (SD: 12.7 years; median: 31.0 years; range: $18.0-78.0$ years $), 51.6 \%(n=99)$ were women. Among the respondents, TSC was diagnosed at a mean age of 10.4 years (SD: 14.9 years; median: 2.0 years; range: 0-66.0 years), and the first symptoms of TSC were noted at a mean age of 5.7 years (SD: 12.0 years; median: 0 years; range: $0-66.0$ years). In three individuals $(1.6 \%)$, a diagnosis of TSC was suspected before birth, based on ultrasound examinations. Pathogenic variants in TSC1 were reported by 32 individuals (16.7\%), and pathogenic variants in TSC2 were reported by 48 individuals $(25.0 \%$, ratio $1: 1.5)$. Three individuals (1.6\%) suffered from a polycystic kidney disease with tuberous sclerosis (PKDTS) contiguous gene deletion syndrome.

Most individuals lived with others. Of those, 44 (22.9\%) were married or in a relationship, and 84 (43.8\%) lived with relatives. Less than half of individuals were either employed $(n=71,37.0 \%)$ or participated in vocational training $(n=21,10.9 \%)$. Further sociodemographic and clinical characteristics, including information on affected family members, are presented in Table 1 . The majority of individuals suffered from a range of TSC organ manifestations. Disorders of the central nervous system were commonly reported, with 140 (72.9\%) individuals reporting a diagnosis of epilepsy, 129 (67.2\%) describing various structural brain disorders, and 97 (50.5\%) indicating psychiatric disorders. Furthermore, 182 (94.8\%) individuals 
Table 1 Sociodemographic and clinical characteristics of participants $(n=192)$

\begin{tabular}{|c|c|}
\hline & All patients $n=192$ \\
\hline \multirow[t]{3}{*}{ Age in years ${ }^{1}$} & $33.4 \pm 12.7$ \\
\hline & Range \\
\hline & 18.0-78.0 \\
\hline Sex & $\%(n)$ \\
\hline Male & $48.4(93)$ \\
\hline Female & $51.6(99)$ \\
\hline \multirow[t]{3}{*}{ Age at first symptoms due to TSC ${ }^{1}$} & $5.7 \pm 12.0$ \\
\hline & Range \\
\hline & $0.0-66.0$ \\
\hline \multirow[t]{3}{*}{ Age at TSC diagnosis in years ${ }^{1}$} & $10.4 \pm 14.9$ \\
\hline & Range \\
\hline & $0.0-66.0$ \\
\hline TSC diagnosis before birth by ultrasound & $\%(n)$ \\
\hline No & $96.9(186)$ \\
\hline Yes & $1.6(3)$ \\
\hline Genetics & $\%(n)$ \\
\hline TSC1-gene & $16.7(32)$ \\
\hline TSC2-gene & $25.0(48)$ \\
\hline TSC2/PKD1 contiguous-gene & $1.6(3)$ \\
\hline No genetic test & $30.7(59)$ \\
\hline No genetic mutation & $10.4(20)$ \\
\hline Unknown & $15.6(30)$ \\
\hline Affected family members by TSC & $\%(n)$ \\
\hline No & $77.6(149)$ \\
\hline Yes & $18.8(36)$ \\
\hline Mother affected ( 43.9 years) ${ }^{2}$ & $4.7(9)$ \\
\hline Father affected $(46.3 \text { years })^{2}$ & $3.6(7)$ \\
\hline Sibling affected $(4.8 \text { years })^{2}$ & $6.8(13)$ \\
\hline Own children affected $(3.0 \text { years })^{2}$ & $8.9(17)$ \\
\hline \multirow[t]{2}{*}{ Number of own affected children } & Mean \\
\hline & 1.4 \\
\hline Marital status & $\%(n)$ \\
\hline Married/living in relationship & $22.9(44)$ \\
\hline Divorced & $1.6(3)$ \\
\hline Single, lives with relatives & $43.8(84)$ \\
\hline Single, lives alone & $29.7(57)$ \\
\hline Unknown/Other & $2.1(4)$ \\
\hline School education & $\%(n)$ \\
\hline$<12$ years & $42.7(82)$ \\
\hline$>12$ years & $20.8(40)$ \\
\hline Still going to school & $4.7(9)$ \\
\hline No school graduation & $30.2(58)$ \\
\hline Not answered & $1.6(3)$ \\
\hline Highest job qualification & $\%(n)$ \\
\hline Missing & $42.2(81)$ \\
\hline Skilled (manual) & $23.4(45)$ \\
\hline Office-based (nonmanual) & $5.7(11)$ \\
\hline University degree & $9.9(19)$ \\
\hline In training & $8.3(16)$ \\
\hline
\end{tabular}

Table 1 (continued)

\begin{tabular}{ll}
\hline & All patients $\mathbf{n}=\mathbf{1 9 2}$ \\
\hline Unknown/Other & $10.4(20)$ \\
Employment situation & $\%(\mathrm{n})$ \\
Employed & $37.0(71)$ \\
Vocational training & $10.9(21)$ \\
Unemployed & $21.4(41)$ \\
Homemaker/parental leave & $1.0(2)$ \\
Early retirement & $9.9(19)$ \\
Old-age pension & $1.0(2)$ \\
Unknown/Other & $18.8(36)$ \\
\hline
\end{tabular}

${ }^{1}$ Mean \pm standard deviation

${ }^{2}$ Mean age at TSC diagnosis of affected family members

reported skin manifestations, 142 (74\%) described kidney and urinary tract disorders, and 97 (50.5\%) indicated heart and circulatory system disorders. Additional details can be found in Table 2 .

\section{Direct costs}

Mean total direct costs were calculated at EUR 6452 (median EUR 1920; 95\% CI EUR 5533-7422) per study participant for the 3-month study period, and details are presented in Table 3 and Fig. 1. Direct medical costs were primarily associated with the costs of drug treatments $(76.8 \%$ of total direct costs; mean EUR 4953 per 3 months; median EUR 573; 95\% CI EUR 4087-5876), and hospitalization (8.0\% of total direct costs; mean EUR 518; median EUR 0; 95\% CI EUR 312-750).

The largest medication costs were those due to mTOR inhibitors (everolimus, $\mathrm{n}=69$; sirolimus, $\mathrm{n}=2$ ), with a mean of EUR 4358 per 3 months (67.5\% of total direct costs; median EUR 0; 95\% CI EUR 3448-5342). mTOR inhibitor costs were higher than those associated with ASDs, which were on average EUR 415 (6.4\% of total direct costs; median EUR 104; 95\% CI EUR 239-706). Individuals used on average 1.9 ASDs (SD: 0.8; median 2: range 1-4). The five most frequently prescribed ASDs were lamotrigine $(n=51 ; 26.6 \%)$, valproate $(n=46$, $24.0 \%)$ oxcarbazepine $(\mathrm{n}=32 ; 16.7 \%)$, levetiracetam $(\mathrm{n}=25 ; 13.0 \%)$, and lacosamide $(\mathrm{n}=12 ; 6.3 \%)$. Monotherapy with ASDs was prescribed to $24.0 \%(n=46)$ of all participants, which was associated with significantly lower costs than polytherapy with two, three, or more ASDs (each $p<0.001$ ). The detailed costs and daily dosages reported for different ASDs are listed in Table 4.

In total, $23(12.0 \%)$ individuals reported at least one TSC-related hospital admission during the 3-month study period. Overall, 29 admissions were reported, with a mean length of stay of 5.5 days (SD: 3.6; median: 5 days; range: $1-14$ days). Epilepsy and seizures resulted 
Table 2 Organ manifestations in individuals with TSC $(n=192)$

\begin{tabular}{|c|c|c|}
\hline & $\%$ & $\mathrm{n}$ \\
\hline Epilepsy & 72.9 & 140 \\
\hline Recurrent seizures & 39.1 & 75 \\
\hline Seizure free $>1$ year or no seizures & 60.9 & 117 \\
\hline Structural brain disorders & 67.2 & 129 \\
\hline Cortical tubers & 49.0 & 94 \\
\hline SEGA ${ }^{1}$ & 37.5 & 72 \\
\hline Hydrocephalus & 2.6 & 5 \\
\hline Psychiatric disorders & 50.5 & 97 \\
\hline Heart and circulatory system & 50.5 & 97 \\
\hline Hypertension & 26.6 & 51 \\
\hline Rhabdomyomas & 24.5 & 47 \\
\hline Arrhythmia & 8.3 & 16 \\
\hline Kidney and urinary tract & 74.0 & 142 \\
\hline Angiomyolipomas & 59.4 & 114 \\
\hline Cysts & 42.2 & 81 \\
\hline Chronic kidney dysfunction & 12.5 & 24 \\
\hline Renal cell carcinoma & 2.6 & 5 \\
\hline Skin manifestations & 94.8 & 182 \\
\hline Angiofibromas & 84.9 & 163 \\
\hline Hypomelanotic macules & 57.3 & 110 \\
\hline Shagreen patches & 48.4 & 93 \\
\hline Ungal/periungal fibromas & 10.9 & 21 \\
\hline Skin tags & 3.6 & 7 \\
\hline Café au lait spots & 2.6 & 5 \\
\hline Lymphangioleiomyomatosis & 11.5 & 22 \\
\hline Other disorders & 39.1 & 75 \\
\hline $\begin{array}{l}\text { Iris or retinal hamartomas/astrocytomas and other } \\
\text { eye disorders }\end{array}$ & 28.6 & 55 \\
\hline Angiomyolipomas in other organ systems ${ }^{2}$ & 14.1 & 27 \\
\hline Cysts in other organ systems ${ }^{2}$ & 13.0 & 25 \\
\hline
\end{tabular}

${ }^{1}$ Subependymal giant cell astrocytoma

${ }^{2}$ Hormone system, Thyroid, Gastrointestinal, Liver, Spleen, Pancreas

in eight admissions, whereas six admissions were associated with diagnostic procedures, four admissions were due to pneumothorax, three were related to operations concerning AML in the kidneys and other organs. A further two admissions were due to adverse reactions to everolimus intake and two were associated with facial skin treatments. Four admissions had other TSC-related reasons.

Ancillary treatments, such as occupational therapy, physiotherapy, and speech therapy, were prescribed to 54 participants $(28 \%)$, and were associated with an average cost of EUR 125 per 3 months, which comprised 1.9\% of total direct costs (median: EUR 0; 95\% CI: EUR 84-174). In addition, families directly paid EUR 39 in therapy-related costs during the 3-month study period.

\section{Care needs and care grade as approximators of informal care costs}

Fifty percent $(n=97)$ of individuals were categorized as requiring care grade levels $\mathrm{I}$ to $\mathrm{V}$, based on the "Pflegebedürftigkeit" scale: $2.6 \%$ as level I ('low impairment of independence'); $9.9 \%$ as level II ('significant need for care'); $11.5 \%$ as level III ('heavy need for care'); $13.5 \%$ as level IV ('most difficult to care for'); and $13 \%$ as level V ('most difficult to care for and special demands regarding nursing care'). Two individuals did not meet the level I-V criteria but were still in need of care according to their caregivers, and $48.4 \%$ of individuals denied being in need of care. The mean approximate costs for nursing care were EUR 929 (median: EUR 0; 95\% CI: EUR 780-1083) over each 3-month period, or EUR 3716 annually, assuming that care is provided by family members. Patient's caregivers reported that they had paid additional costs for care, with a mean of EUR 24 (median; EUR 0; 95\% CI: EUR 10-41). Furthermore, they paid for supervision, with a mean of EUR 48 (median: EUR 0; 95\% CI: EUR 22-79) per 3-month period. Further informal care costs that were neither reflected in the care grade allowance nor perceived by caregivers are inevitably not represented in our approximation of informal care costs. In total, 124 individuals (64.6\%) had a handicapped ID, indicating a degree of disability between 70 and $100 \%$.

\section{Indirect (productivity) costs}

The estimation of mean indirect costs was based only on questionnaire responses from patients of working age, younger than 67 years $(n=190)$. The mean total indirect costs were EUR 3174 (median: EUR 0; 95\% CI: 2503-3840) over three months or EUR 12,696 annually. The main contributor to indirect costs $(n=30)$ was the inability to work due to intellectual disability, epilepsy, or kidney disorders (mean: EUR 1775; median: EUR 0; 95\% CI: EUR 1183-2367). Furthermore, 19 individuals were only able to work part-time, which was associated with a mean estimated cost of EUR $514 \pm 1762$ per 3 months (median: EUR 0; 95\% CI: EUR 283-792). Twenty-eight individuals reported missing days from work during the last three months due to TSC-related causes (mean: EUR 234; median: EUR 0; 95\% CI: EUR 115-382), and six individuals were unemployed (mean: EUR 355; median: EUR 0; 95\% CI: EUR 118-651). Five individuals reported retiring prematurely (mean: EUR 296; median: EUR 0; 95\% CI: EUR 59-592). The details of indirect productivity costs can be found in Table 5 and Fig. 1. The mean duration of work absenteeism was $7.6 \pm 10.7$ days (range: $1-50$ days) per 3 months. 
Table 3 Direct costs for the 3-month study period for the total patient group ( $n=192 ;$ in 2019 Euro)

\begin{tabular}{|c|c|c|c|c|c|c|c|c|}
\hline Cost components & Mean costs & $\mathrm{SD}^{1}$ & Minimum & Median & Maximum & $95 \% \mathrm{Cl}$ & $\begin{array}{l}\% \text { of total } \\
\text { direct costs }\end{array}$ & $\begin{array}{l}\text { Estimated } \\
\text { annual direct } \\
\text { costs }^{2}\end{array}$ \\
\hline Total direct costs & 6452 & 7584 & 0 & 1920 & 29,182 & $5533 ; 7422$ & 100 & 25,808 \\
\hline Medication $(n=165)$ & 4953 & 6854 & 0 & 573 & 28,224 & $4087 ; 5876$ & 76.8 & 19,812 \\
\hline mTOR inhibitors* $(n=71)$ & 4358 & 6520 & 0 & 0 & 25,273 & $3448 ; 5342$ & 67.5 & 17,432 \\
\hline Antiseizure drugs (ASDs) $(n=123)$ & 415 & 1962 & 0 & 104 & 26,538 & $239 ; 706$ & 6.4 & 1660 \\
\hline Other prescription drugs $(n=104)$ & 132 & 385 & 0 & 8 & 2606 & $84 ; 186$ & 2.0 & 528 \\
\hline OTC drugs and supplements $(n=70)$ & 41 & 100 & 0 & 0 & 700 & $29 ; 54$ & 0.6 & 164 \\
\hline $\begin{array}{l}\text { Emergency medication/medication } \\
\text { on demand }(n=42)\end{array}$ & 7 & 36 & 0 & 0 & 347 & $3 ; 13$ & 0.1 & 28 \\
\hline Hospitalization $(n=23)$ & 518 & 1691 & 0 & 0 & 11,487 & $312 ; 750$ & 8.0 & 2072 \\
\hline Outpatient treatment $(n=157)$ & 467 & 1156 & 0 & 194 & 15,097 & $352 ; 626$ & 7.2 & 1868 \\
\hline Diagnostics $(n=140)$ & 155 & 242 & 0 & 44 & 1691 & $124 ; 192$ & 2.4 & 620 \\
\hline Ancillary therapies $(n=54)$ & 125 & 307 & 0 & 0 & 2120 & $84 ; 174$ & 1.9 & 500 \\
\hline Auxillary material $(n=14)$ & 49 & 253 & 0 & 0 & 2235 & $18 ; 87$ & 0.8 & 196 \\
\hline Rehabilitation $(\mathrm{n}=2)$ & 40 & 410 & 0 & 0 & 4983 & $0 ; 92$ & 0.6 & 160 \\
\hline Emergency service use $(n=9)$ & 44 & 217 & 0 & 0 & 1800 & $19 ; 75$ & 0.7 & 176 \\
\hline Specific diets $(n=3)$ & 9 & 97 & 0 & 0 & 1200 & $0 ; 23$ & 0.1 & 36 \\
\hline Transport costs $(n=25)$ & 5 & 17 & 0 & 0 & 130 & $3 ; 7$ & 0.1 & 20 \\
\hline Co-payments for therapies $(n=17)$ & 39 & 177 & 0 & 0 & 1400 & $19 ; 64$ & 0.6 & 156 \\
\hline Other co-payments $(n=45)$ & 48 & 183 & 0 & 0 & 1700 & $27 ; 75$ & 0.7 & 192 \\
\hline
\end{tabular}

$95 \% \mathrm{Cl}=95 \%$ Confidence interval using the bootstrap bias corrected and accelerated method ${ }^{1}$ Standard deviation, ${ }^{2}$ Estimation based on the mean costs in three months multiplied by four

*Everolimus $n=69$, Sirolimus $n=2$, OTC $=$ over-the-counter

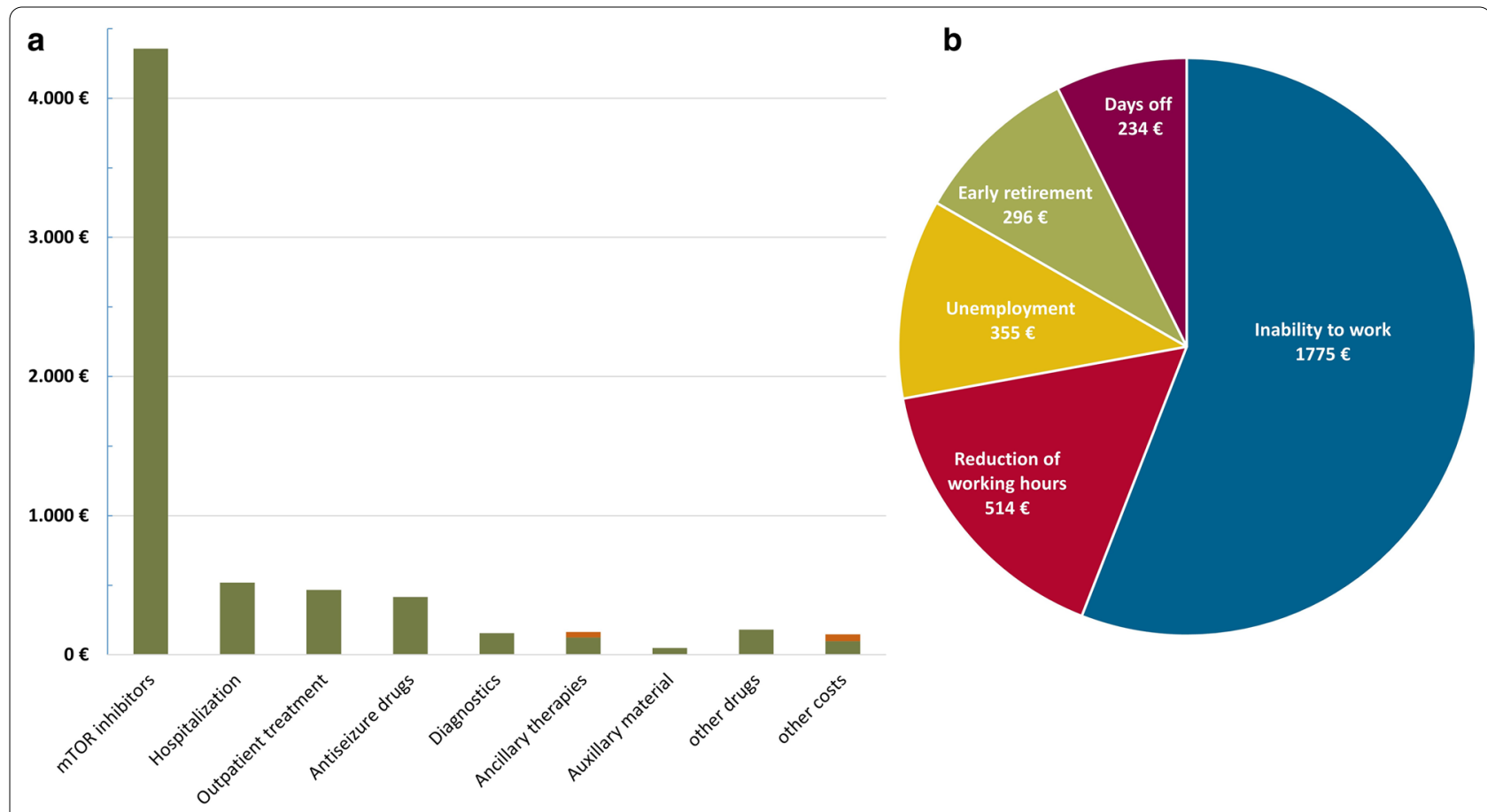

Fig. 1 Breakdown of total direct costs (a), with copayments in orange, per patient over 3 months, and total indirect costs over 3 months (b) 
Table 4 Prescription patterns and costs of antiseizure drugs for the 3-month study period (in 2019 Euro)

\begin{tabular}{|c|c|c|c|c|c|c|c|c|}
\hline Medication costs & $\mathrm{n}$ & $\begin{array}{l}\text { Mean costs per } \\
3 \text { months }\end{array}$ & $S D^{1}$ & Minimum & Median & Maximum & $95 \% \mathrm{Cl}$ & p-value ${ }^{2}$ \\
\hline All patients & 192 & $€ 415$ & 1962 & $€ 0$ & $€ 104$ & $€ 26,538$ & $239 ; 706$ & \\
\hline No ASDs (35.9\%) & 69 & 0 & & & & & & \\
\hline Monotherapy (24.0\%) & 46 & $€ 222$ & 400 & $€ 15$ & $€ 125$ & $€ 2613$ & $136 ; 354$ & $<0.001^{3}$ \\
\hline 2 ASDs (26.0\%) & 50 & $€ 327$ & 317 & $€ 50$ & $€ 238$ & $€ 1405$ & $250 ; 415$ & $<0.010^{4}$ \\
\hline$\geq 3 \operatorname{ASDs}(14.1 \%)$ & 27 & $€ 1965$ & 4979 & $€ 144$ & $€ 962$ & $€ 26,538$ & 826,4016 & $<0.001^{5}$ \\
\hline Prescribed medication & $n$ & Mean daily dose & $\mathrm{SD}^{1}$ & Minimum & Median & Maximum & $\begin{array}{l}\text { Mean costs per } \\
3 \text { months }\end{array}$ & $\mathrm{SD}^{1}$ \\
\hline Lamotrigine (26.6\%) & 51 & $362 \mathrm{mg}$ & $373 \mathrm{mg}$ & $50 \mathrm{mg}$ & $300 \mathrm{mg}$ & $2500 \mathrm{mg}$ & $€ 90$ & 93 \\
\hline Valproate (24.0\%) & 46 & 1355 mg & $465 \mathrm{mg}$ & $450 \mathrm{mg}$ & $1250 \mathrm{mg}$ & $2300 \mathrm{mg}$ & $€ 62$ & 21 \\
\hline Oxcarbazepine (16.7\%) & 32 & $1617 \mathrm{mg}$ & $708 \mathrm{mg}$ & $150 \mathrm{mg}$ & $1800 \mathrm{mg}$ & $3600 \mathrm{mg}$ & $€ 226$ & 99 \\
\hline Levetiracetam (13.0\%) & 25 & $2400 \mathrm{mg}$ & 1090 mg & $750 \mathrm{mg}$ & $2250 \mathrm{mg}$ & $4500 \mathrm{mg}$ & $€ 161$ & 73 \\
\hline Lacosamide (6.3\%) & 12 & $410 \mathrm{mg}$ & $283 \mathrm{mg}$ & $100 \mathrm{mg}$ & $350 \mathrm{mg}$ & $1200 \mathrm{mg}$ & $€ 894$ & 616 \\
\hline Topiramate (3.6\%) & 7 & $225 \mathrm{mg}$ & $56 \mathrm{mg}$ & $150 \mathrm{mg}$ & $200 \mathrm{mg}$ & $300 \mathrm{mg}$ & $€ 149$ & 37 \\
\hline Zonisamide (3.6\%) & 7 & $657 \mathrm{mg}$ & $450 \mathrm{mg}$ & $300 \mathrm{mg}$ & $500 \mathrm{mg}$ & $1600 \mathrm{mg}$ & $€ 1087$ & 745 \\
\hline Carbamazepine (3.1\%) & 6 & $700 \mathrm{mg}$ & $490 \mathrm{mg}$ & $300 \mathrm{mg}$ & $500 \mathrm{mg}$ & $1600 \mathrm{mg}$ & $€ 35$ & 24 \\
\hline Perampanel (3.1\%) & 6 & $5 \mathrm{mg}$ & $2 \mathrm{mg}$ & $2 \mathrm{mg}$ & $6 \mathrm{mg}$ & $8 \mathrm{mg}$ & $€ 308$ & 120 \\
\hline Brivaracetam (2.6\%) & 5 & $220 \mathrm{mg}$ & $76 \mathrm{mg}$ & $150 \mathrm{mg}$ & $200 \mathrm{mg}$ & $350 \mathrm{mg}$ & $€ 508$ & 175 \\
\hline Sulthiame (2.6\%) & 5 & $530 \mathrm{mg}$ & $470 \mathrm{mg}$ & $100 \mathrm{mg}$ & $450 \mathrm{mg}$ & $1250 \mathrm{mg}$ & $€ 320$ & 284 \\
\hline Vigabatrine (2.6\%) & 5 & $2200 \mathrm{mg}$ & $758 \mathrm{mg}$ & $1000 \mathrm{mg}$ & $2500 \mathrm{mg}$ & $3000 \mathrm{mg}$ & $€ 397$ & 137 \\
\hline Phenytoin (2.1\%) & 4 & $331 \mathrm{mg}$ & $85 \mathrm{mg}$ & $250 \mathrm{mg}$ & $313 \mathrm{mg}$ & $450 \mathrm{mg}$ & $€ 26$ & 7 \\
\hline Primidone (2.1\%) & 4 & $750 \mathrm{mg}$ & $451 \mathrm{mg}$ & $375 \mathrm{mg}$ & $625 \mathrm{mg}$ & $1250 \mathrm{mg}$ & $€ 38$ & 23 \\
\hline Phenobarbital (2.1\%) & 4 & $141 \mathrm{mg}$ & 106 mg & $10 \mathrm{mg}$ & $163 \mathrm{mg}$ & $230 \mathrm{mg}$ & $€ 62$ & 47 \\
\hline Rufinamid (1.6\%) & 3 & $1267 \mathrm{mg}$ & $702 \mathrm{mg}$ & $600 \mathrm{mg}$ & $1200 \mathrm{mg}$ & $2000 \mathrm{mg}$ & $€ 759$ & 421 \\
\hline Clobazame (1.6\%) & 3 & $25 \mathrm{mg}$ & $13 \mathrm{mg}$ & $15 \mathrm{mg}$ & $20 \mathrm{mg}$ & $40 \mathrm{mg}$ & $€ 68$ & 36 \\
\hline Gabapentin (1.6\%) & 3 & $967 \mathrm{mg}$ & $1250 \mathrm{mg}$ & $100 \mathrm{mg}$ & $400 \mathrm{mg}$ & $2400 \mathrm{mg}$ & $€ 78$ & 101 \\
\hline Other ASDs* $(5.7 \%)$ & 11 & & & & & & & \\
\hline
\end{tabular}

${ }_{1}$ Standard deviation, $95 \% \mathrm{Cl}=95 \%$ Confidence interval using the bootstrap bias corrected and accelerated method

${ }^{2}$ Mann-Whitney-U-test; ASD = antiseizure drug; ${ }^{3}$ Monotherapy vs. $\geq 3$ ASDs, ${ }^{4}$ Monotherapy vs. 2 ASDs ${ }^{5} 2$ ASDs vs $\geq 3$ ASDs,

*(Cannabidiol $n=2$, Clonazepam $n=2$, Ethosuximide $n=2$, Lorazepam $n=2$, Mesuximide $n=1$, Pregabalin $n=2$ )

Table 5 Indirect costs for individuals with TSC during the 3-month study period (in 2019 Euro)

\begin{tabular}{|c|c|c|c|c|c|c|c|c|}
\hline Indirect costs components & $n^{1}$ & Mean costs & $\mathrm{SD}^{2}$ & Minimum & Median & Maximum & $95 \% \mathrm{Cl}$ & $\begin{array}{l}\text { Estimated } \\
\text { annual } \\
\text { costs }^{3}\end{array}$ \\
\hline Total indirect costs $(<67 \mathrm{y})$ & 86 & 3174 & 4703 & 0 & 0 & 11,241 & $2503 ; 3840$ & 12,696 \\
\hline Inability to work & 30 & 1775 & 4110 & 0 & 0 & 11,241 & $1183 ; 2367$ & 7100 \\
\hline Reduction of working hours & 19 & 514 & 1762 & 0 & 0 & 9695 & $283 ; 792$ & 2056 \\
\hline Unemployment & 6 & 355 & 1971 & 0 & 0 & 11,241 & $118 ; 651$ & 1420 \\
\hline Early retirement & 5 & 296 & 1804 & 0 & 0 & 11,241 & $59 ; 592$ & 1184 \\
\hline Days off & 28 & 234 & 1050 & 0 & 0 & 10,750 & $115 ; 382$ & 936 \\
\hline
\end{tabular}

95\% $\mathrm{Cl}=95 \%$ Confidence interval using the bootstrap bias corrected and accelerated method

${ }^{1}$ Patients of working age $(n=190),{ }^{2}$ Standard deviation, ${ }^{3}$ Estimation based on the mean costs in three months multiplied by four

Cost drivers of direct, indirect, and approximated informal (nursing) care costs

To identify potential cost drivers, we performed univariate analyses for total direct, total indirect, and nursing care costs and a number of demographic and clinical characteristics. For details, please refer to Table 6. 


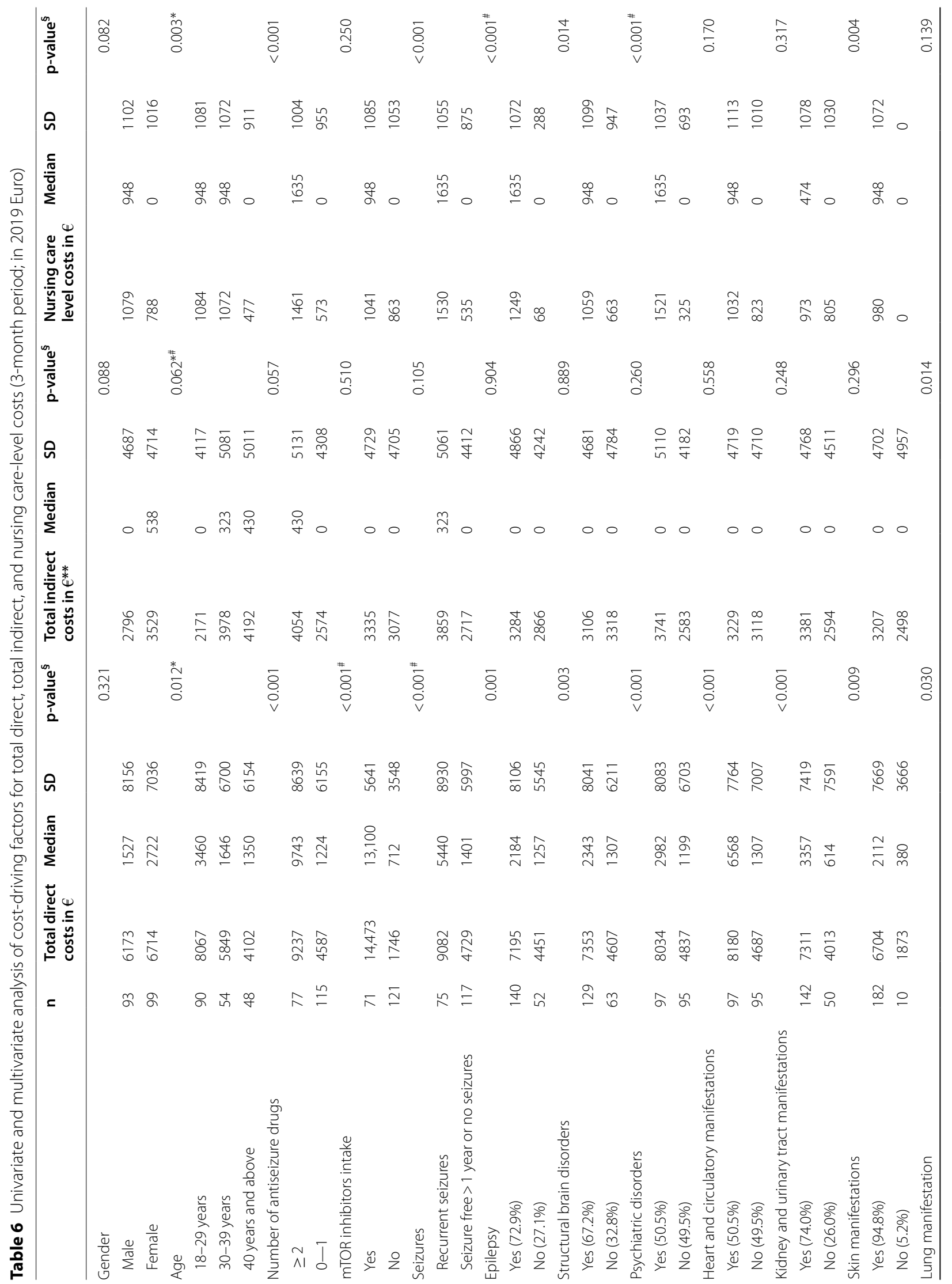




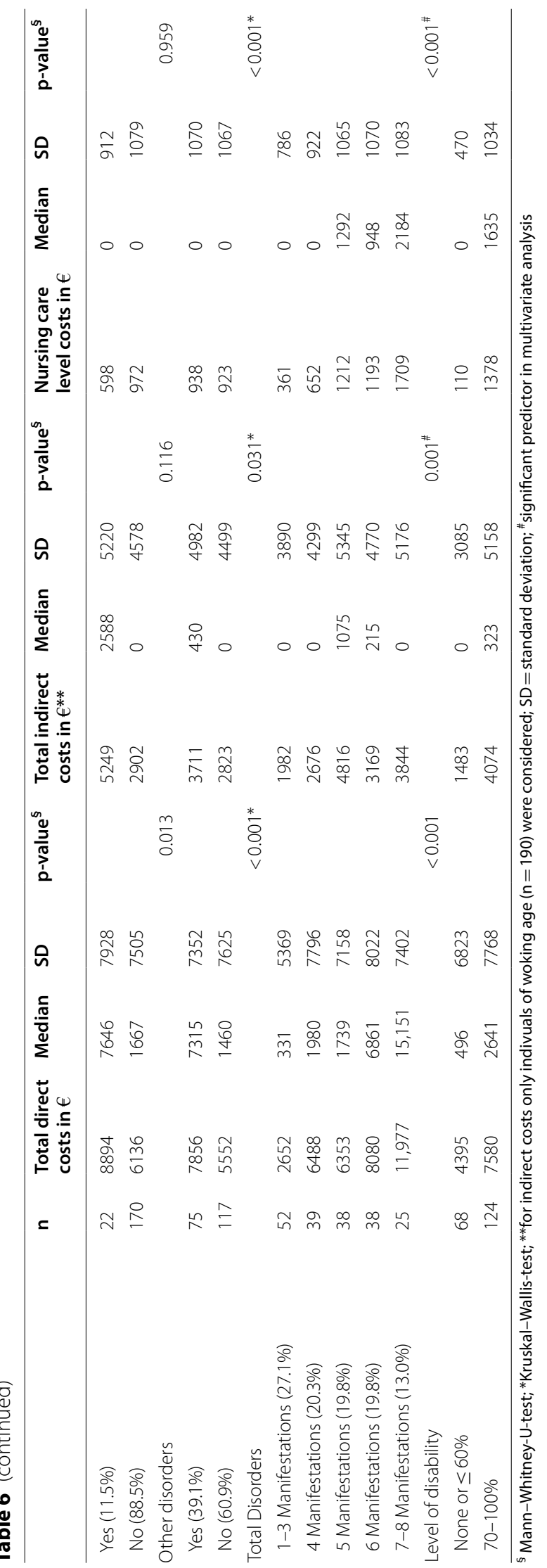


In the univariate analyses, younger age, the use of mTOR inhibitors, polytherapy with two or more ASDs, recurrent seizures, all TSC manifestation categories, the total number of TSC manifestations, and the level of disability were associated with higher direct costs. Lung manifestations (lymphangioleiomyomatosis), the total number of TSC manifestations, and disability were associated with higher indirect costs, whereas younger age, polytherapy with two or more ASDs, recurrent seizures, the TSC manifestations of epilepsy, structural brain disorders, psychiatric disorders, and skin manifestations, the total number of manifestations, and disability were associated with increased nursing care costs. Overall, total direct, indirect, and nursing costs increased with the number of affected organ systems (Table 6).

Multiple linear regression analyses revealed that the use of mTOR inhibitors independently predicted a 3-month direct cost increase of 12,069 Euro (BCa-corrected $\mathrm{B}$ 12,068.85, BCa-corrected standard error [SE] B 836.28, $\beta 0.770, p<0.001$ ), and persistent seizures predicted a 3-month direct cost increase of 2113 Euro (B 2113.29, SE B 651.13, $\beta$ 0.137, $p<0.001$ ). Applying a Bonferroni correction for twelve comparisons, the threshold for the p-value was set at 0.00417 , and the mTOR inhibitor use and persistent seizures were able to explain $71 \%$ $\left(R^{2}, \mathrm{~F}(12,179)=37.09, \mathrm{~F}\right.$ sig. $)$ of the total direct cost variance. Older age and disability were independent costdriving factors for total indirect costs, with disability predicting a 3-month indirect cost increase of 2131 Euro (B 2131.48, SE B 693.90, $\beta 0.216, p=0.004$ ) and older age of 1220 Euro per 3 months (B 1220.05, SE B 391.20, $\beta 0.212$, $p=0.003)$. The significant factors together explained $13 \%$ of the indirect cost variance (corrected $p<0.0125$; $R^{2}=13 \%$, F $(5,184)=5.67$, F sig.). Epilepsy, psychiatric diseases, and disability were independent cost drivers for approximate informal (nursing) care costs, with relatively similar cost-driving effects. Disability predicted a 3-month nursing-cost increase of 622 Euro (B 622.02, SE B 160.86, $\beta$ 0.280, $p<0.001)$, psychiatric diseases of 599 Euro (B 599.45, SE B 149.67, $\beta$ 0.282, $p<0.001$ ), and epilepsy of 528 Euro (B 528.27, SE B 138.67, $\beta$ 0.221, $p=0.001$ ). Together, these significant variables (corrected $p<0.00625)$ were able to explain $51 \%$ of the 3 -month nursing care cost variance $\left(R^{2}=51 \%, \mathrm{~F}(9,182)=20.98\right.$, F sig.).

\section{Discussion}

This detailed, multicenter, COI study is based on a large sample of 192 adult individuals with TSC within a single healthcare system and contributes important new information about the direct and indirect costs and related cost drivers associated with TSC in Europe. To enable comparisons with other COI studies, we aimed to capture the most comprehensive set of cost items related to epilepsy and other TSC organ manifestations [4, 39]. Previous studies have reported direct cost estimates for individuals with TSC in Europe [15, 16, 40, 41], North America [9, 11, 37, 38, 42-44], and Asia [45], but none of these previous studies have provided indirect cost estimates for adults affected by TSC or examined the cost drivers of indirect and nursing care costs [4].

\section{Direct costs and related cost drivers}

Our study highlights the substantial direct costs incurred by individuals with TSC. Medication was the largest single component of direct costs and associated with an estimated annual direct cost of EUR 25,808. The highest medication costs were due to mTOR inhibitors (annual costs of EUR 17,432), which were used by 37\% (71/192) of the individuals in this study. mTOR inhibitor use was identified as an independent cost-driver in the multivariate analysis. This finding is somewhat expected, given the currently high price of mTOR inhibitors. Everolimus, the mTOR inhibitor overwhelmingly used by individuals in this study, was first given conditional marketing authorization as an orphan drug by the European Medicines Agency (EMA) in 2011 for TSC-associated SEGA and in 2012 for TSC-associated renal AML [46], followed by an extension of indication to epilepsy refractory to ASD in 2017. Due to the time point of this study, the long-term cost-effectiveness of this currently costly drug remains to be evaluated [47]. The possibility of avoiding potentially costly TSC consequences, such as resection surgery for SEGA or epilepsy, cerebral shunt placement, and AMLassociated renal bleeding following everolimus may balance favorably against the unwanted treatment effects of mTOR inhibitors, and everolimus may emerge as a costeffective treatment option. The costs associated with the use of mTOR inhibitors will also likely decrease in the future due to the availability of generic formulations.

Interestingly, gender differences were not associated with differences in TSC-related costs. Women with TSC are known to be more likely to develop AML, and AML in women tends to be larger and require more interventions [10]. Women are also almost exclusively affected by pulmonary manifestations of TSC, which have been demonstrated to incur high direct costs [11, 15]. The findings in the present study may be due to the relative rarity of severe LAM complications, which typically occur only with increasing age. However, we did record four hospital admissions among our cohort for pneumothorax, a known complication of LAM, within the short evaluation period of three months. An alternative explanation is that the salient contributions of mTOR inhibitor therapy to overall costs, which exceeded inpatient treatment costs, may have 
masked gender differences in our cohort. Young adults (18-29 years) with TSC incurred higher costs than older individuals did, which is likely associated with the performance of a larger proportion of diagnostic procedures and the increasing use of mTOR inhibitors among younger patients.

In adults, renal manifestations of TSC are more common than in children. The most common types of renal manifestations, including AML and renal cysts, tend to appear first during adolescence and grow during adulthood. In our study, a similar proportion of individuals reported AML as in the TOSCA cohort [10], and renal manifestations were a significant factor for direct costs in the univariate analysis. Interestingly, in the multivariate analysis, only recurring seizures remained an independent clinical cost driver, and no single other TSC manifestation category was identified as an independent cost driver, which may be due to the higher direct costs associated with recurrent seizures compared with all of the other seven clinical categories, which were all associated with similar direct costs. This finding supports the known severe burden of illness of ongoing epilepsy in TSC, particularly because a relevant share of individuals experience pharmacorefractory epilepsy [48]. The high direct costs are in line with those reported for other rare developmental and epileptic encephalopathies like Dravet syndrome [49] or Lennox-Gastaut-syndrome [50]. This finding further highlights the need for ongoing identification of epilepsy surgical candidates among those with TSC and epilepsy, new emerging therapies such like the MR-guided laser interstitial thermal therapy might help to increase the suitability of patients for a surgical treatment [51]. Generally, the results of this study indicate that the management of TSC might result in high direct costs that exceed the costs incurred by all-cause epilepsy patients [26]. Our multivariate analysis model was able to account for $71 \%$ of the total variance in directs costs, suggesting that mTOR inhibitor use together with recurring seizures can explain a relevant share of the direct cost components among TSC patients in Germany. Similar to results reported for the United Kingdom [15], direct costs increased with the number of TSC manifestations. Although this finding was expected due to the complexity of TSC, which necessitates surveillance and treatment for most manifestations, this finding further demonstrated the need for systemic causal treatment and integrated, streamlined care, such as by specialized TSC centers.

Cannabidiol (CBD) is a new treatment option for drugresistant seizures associated with TSC, it has recently been approved by the United States Federal Drug Administration (FDA) and the EMA. CBD has shown promising results in a randomized-controlled study published recently [52]. Since our study preceded the approval of
CBD for TSC in the European Union, only two patients were treated with CBD in our cohort and the influence of $\mathrm{CBD}$ on the direct cost is negligible in our results.

Most cost categories were heavily skewed due to the clinical heterogeneity among adult individuals with TSC. While a few patients required significant health care resources, many were only mildly affected. In our study, the ratio of patients diagnosed with TSC1 vs. TSC2 mutations was slightly higher than anticipated from past study findings $[12,53]$. Because individuals with TSC2 mutations tend to be more severely affected, especially by neuropsychiatric manifestations and epilepsy, an even higher COI can be presumed among populations with higher shares of TSC2 mutations. However, we caution that a large proportion of the patients in this study did not report any genetic test results.

\section{Indirect costs and related cost drivers}

To our knowledge, this is the first study to report the indirect costs of TSC. Nearly half of individuals (86/192, 46\%) who participated in this study reported productivity losses. Adult individuals with TSC in our study incurred substantial indirect annual costs, equal to EUR 12,696 per year, with the largest share due to the inability to work (EUR 7100). Approximately two-thirds (132/192, 67\%) of individuals in our study were able to work without impairment (excluding extra days off due to TSC). The indirect costs reported in our study are broadly similar to those reported for other rare neurological diseases, such as spinal muscular atrophy [54] or Becker's muscular dystrophy [55], but were substantially higher than those reported among individuals with all-cause epilepsy [24]. Unlike direct costs, only a few clinical or demographic categories were associated with increased indirect costs on univariate analysis. We identified both the manifestation burden and the level of disability as associated with increased indirect costs. LAM also emerged as a variable significantly associated with higher indirect costs, indicating that while rare overall, LAM may play an outsize role in affected individuals due to its associated severe impairments.

Incomplete participation due to part-time work, unemployment, and early retirement was common even among those who were able to participate in the primary work market. Additionally, TSC impairs participation in education, as demonstrated by the high proportion of individuals that did not report any job qualifications (42.2\%) and that did not graduate from school (30.2\%). In addition, half of patients required care on the care grade allowance scale. Most clinical variables were not independently associated with higher approximated informal (nursing) care costs in the multivariate analysis, e.g. heart manifestations (which was primarily arterial hypertension 
among this adult cohort), renal manifestations, and lung manifestations. These types of clinical manifestations are typically intermittent, as with AML bleeding, or mostly do not necessitate nursing care, such as renal or pulmonary insufficiency. In contrast, epilepsy, neuropsychiatric manifestations, and disability were independent cost drivers that frequently required nursing care. We must caution that our approach of assessing informal care costs from allowances according to care grades necessarily misses all intangible informal costs that were not perceived by the caregiver as further costs and thus not specifically reported. This includes e.g. losses of caregiver productivity due to providing informal (= not by a health professional) care. True informal care costs are thus most likely higher than in our approximation.

\section{Comparison with earlier studies}

The direct costs identified in our study are broadly comparable to those reported by other recent studies (see Table 7 for a comparison of costs). However, costs in our cohort were both higher than the annual direct costs reported by studies from the UK (GBP 4227-5054 per year) $[15,16,41]$ and lower than the costs reported by two studies from the US (USD 8543-85,397 per year) [37, 38]. The direct costs associated with ASD were lower in our study than those reported in previous studies, despite the specific drugs that were reported being similar [44]. This finding likely reflects lower medication costs in Germany due to price negotiations between statutory health insurers and drug manufacturers in recent years [56]. Importantly, this finding of lower costs was not true for mTOR inhibitors, which incurred higher costs than were reported by another study from the US [44]. The costs associated with hospitalizations and outpatient treatment were lower in our study than in earlier studies. Interestingly, ancillary therapies represented a smaller share of total direct costs than were reported in another study that explicitly reported this variable [43], which is likely due to differences in the reimbursement policies between varying healthcare systems. In general, direct comparisons to studies from different settings or countries are difficult because due to differing definitions, policies, measurements and other factors. To date, only one other study has analyzed productivity losses in adults with TSC [43]. Interestingly, although this study was performed in a different country, activity impairment was similar, with approximately one-third of adult individuals with TSC reporting not fully participating in the work market.

In terms of the use of healthcare resources, our results appear to be in line with other COI studies, particularly hospitalization frequency and the use of ASDs $[9,11,15$, $16,37,42,44,45]$. Most studies could not evaluate mTOR inhibitor use because the periods of data inclusion preceded their authorization. In our study, more patients (37\%) used mTOR inhibitors than in three other recent studies $(8-16.5 \%)$ [37, 44, 45].

A particular contribution of this study is the collection of data regarding the indirect costs and the nursing requirements, which were measured by the care grade allowances. Nursing-associated costs were identified as an important cost component, associated with annual expenditures of EUR 3716, reinforcing the importance of different organ manifestations and seizure-related costs, as were reported by Skalicky et al. [43].

\section{Limitations}

The limitations associated with the questionnaire used in this study include the potential for recall bias regarding three-month-old events, which might result in incomplete and underestimated costs. Furthermore, although the sample consisted of individuals recruited from a variety of sources (multiple clinics and centers across Germany and through the patient advocacy group), we do not know whether the included sample is representative of individuals with TSC in Germany owing to the rarity of TSC. Only two individuals older than 67 years of age were included in this study, which may indicate the limited access to specialized care among this vulnerable group. In addition, the analysis of cost drivers should be interpreted with caution given the limited sample size. However, the significance that the number of organ manifestations had on COI in the current study suggests some common ground with earlier studies, which also identified the number of organ manifestations as cost driver [16]. In addition, the skewness identified in the cost calculations should be noted, as disparities were noted between the mean and median costs. A limitation is the incomplete capturing of informal care costs, as we based these only on allowances paid to the individual for support of caregivers, and services that were paid outof-pocket by the patients or caregivers. Further intangible and likely substantial informal care costs such as work productivity loss of caregivers is explicitly exempt from our analysis. Another limitation of the study was due to the calculation of indirect costs using the human capital approach. The study was performed in 2019 in a situation of nearly full employment in the general population before the onset of the Corona virus disease (COVID-19) pandemic, however indirect costs may not exactly reflect the burden on society and may be overestimated [57]. However, due to the limitations of the friction cost approach [58], we retained the human capital approach, which is in accordance with the German and international recommendations for performing health economic evaluations [59-61]. The major strength of the study remains its large sample size of 192 individuals 


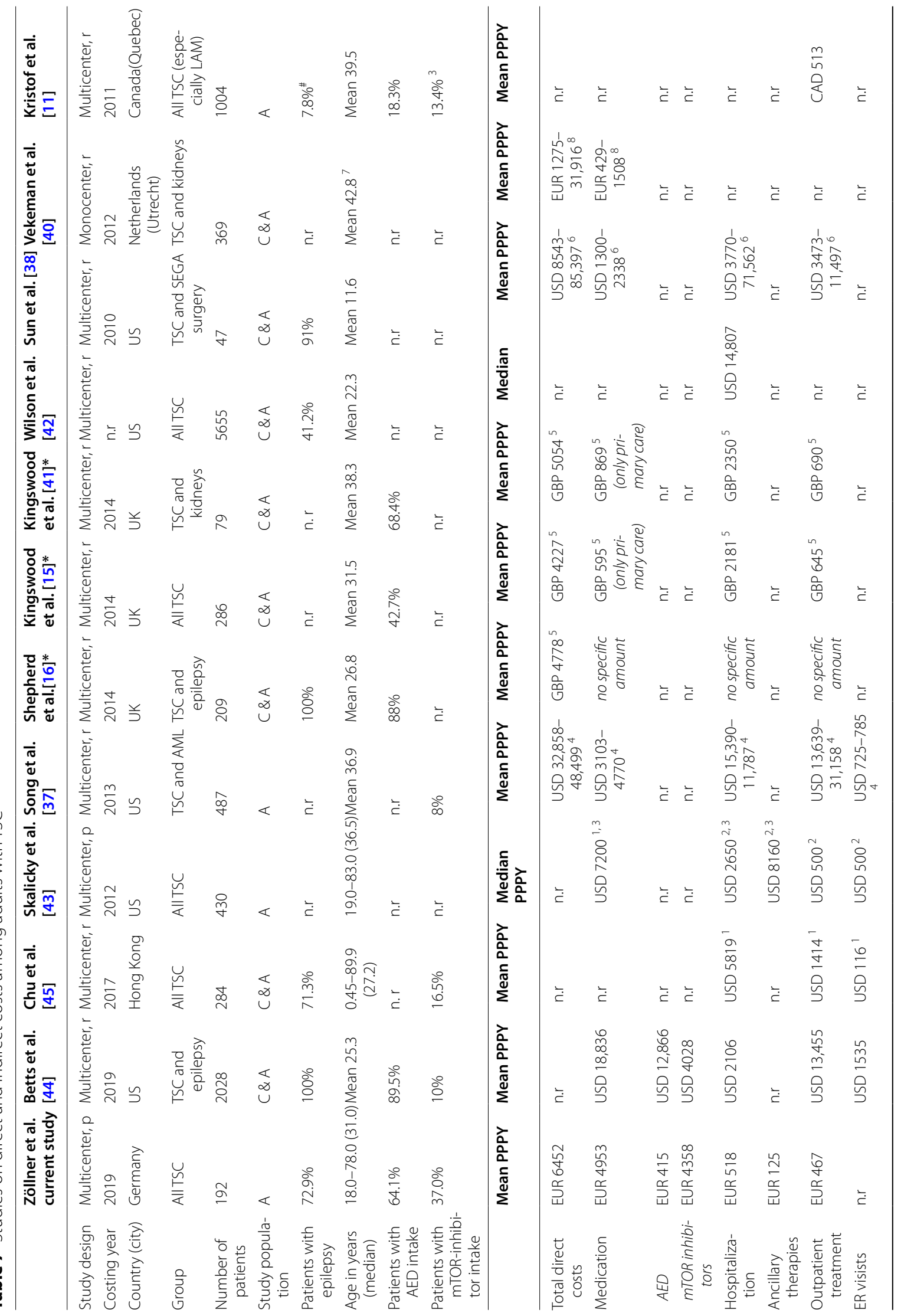




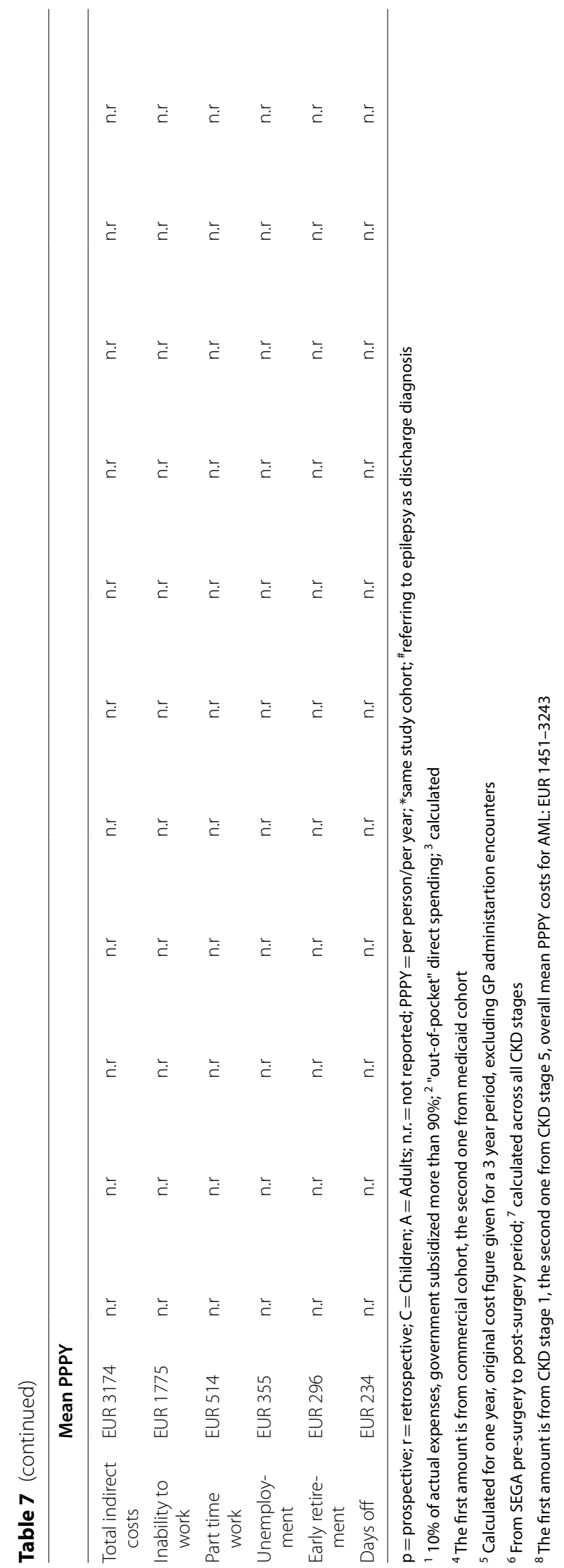


and caregivers, which is significant given the rarity of TSC, and the timing of this analysis after mTOR inhibitors were licensed for the treatment of various disease manifestations.

\section{Conclusions}

Expenditures among individuals with TSC are high and are driven by the number and severity of disease manifestations. More effective delivery of existing disease-modifying treatments and the development of new therapies may have the potential to substantially reduce the high clinical and economic burden of TSC for patients and our health system. Productivity losses represent a major source of costs, which should be addressed through improved sociomedical support and therapeutic interventions. Efforts should focus on reducing absenteeism from work and providing integrated, centralized care for individuals with TSC.

\section{Supplementary Information}

The online version contains supplementary material available at https://doi. org/10.1186/s13023-021-01838-w.

Additional file 1. Methods of cost and price calculation.

\section{Acknowledgements}

The authors would like to thank all of the individuals with TSC who contributed to this survey and the German patient advocacy group Tuberöse Sklerose Deutschland e.V. for their help with participant recruitment.

\section{Authors' contributions}

$J P Z, J G, F R, M S, G K, C H, M K, S S B$, and AS developed the idea for this study. $J P Z$, JG, and AS conceived the paper, collected the data, and performed the statistical analysis. JG and AS created the charts and figures. JPZ, JG, and AS performed the review of cost-of-illness studies. All authors wrote the paper, discussed the results, contributed to the final manuscript, and approved the final manuscript for publication.

\section{Funding}

Open Access funding enabled and organized by Projekt DEAL. This study was supported by the State of Hessen with a LOEWE-Grant to the CePTER Consortium (https://www.uni-frankfurt.de/67689811).

\section{Availability of data and materials}

The datasets analyzed during the current study are available from the corresponding author on reasonable request.

\section{Declarations}

\section{Ethics approval and consent to participate}

This study received ethics approval by the Goethe-University Frankfurt (reference 324/18), and all participants provided informed consent.

\section{Consent for publication}

Not applicable.

\section{Competing interests}

JPZ reports speakers' honoraria and travel grants from EISAI and Desitin Arzneimittel. FR reports personal fees from Arvelle Therapeutics, Desitin Arzneimittel, Eisai, GW Pharmaceuticals companies, Novartis, Medtronic, Sandoz, Shire, and UCB, and grants from the Detlev-Wrobel-Fonds for Epilepsy Research, the Deutsche Forschungsgemeinschaft, the LOEWE Programme of the State of Hesse, and the European Union. MS reports personal fees from Novartis and GW Pharmaceuticals companies. GK reports personal fees from Desitin Arzneimittel, Eisai, GW Pharmaceuticals companies, UCB, Novartis, Takeda, and Zogenix. TM reports personal fees and grants from Arvelle Therapeutics, Eisai, GW Pharmaceuticals companies, UCB, and Zogenix. AB reports personal fees from Desitin Arzneimittel $\mathrm{GmbH}$, Eisai $\mathrm{GmbH}$, Eisai $\mathrm{GmbH}$, Shire $\mathrm{GmbH}$, UCB Pharma GmbH, and ViroPharma GmbH. KMK reports personal fees from UCB Pharma, Novartis Pharma AG, Eisai, and GW Pharmaceuticals, grants from the federal state Hessen through the LOEWE program, and from the Canadian Institutes of Health Research. SM reports grants from Novartis, UCB, Shire, Deutsche Forschungsgemeinschaft and Epilepsiestiftung Dr. Wolf. FvP reports personal fees and grants from Bial, Desitin Arzneimittel, Eisai, GW Pharmaceutical companies, Arvelle Therapeutics, and UCB Pharma. SS-B reports personal fees from Eisai, Desitin Pharma, GW Pharmaceuticals companies, LivaNova, UCB, and Zogenix. AS reports personal fees and grants from Arvelle Therapeutics, Desitin Arzneimittel, Eisai, GW Pharmaceuticals companies, LivaNova, Marinus Pharma, Medtronic, UCB, and Zogenix. None of the other authors reported any related conflicts of interest.

\section{Author details}

${ }^{1}$ Epilepsy Center Frankfurt Rhine-Main, Department of Neurology, GoetheUniversity Frankfurt, Schleusenweg 2-16, 60528 Frankfurt am Main, Germany. ${ }^{2}$ Center for Personalized Translational Epilepsy Research (CePTER), GoetheUniversity Frankfurt, Frankfurt am Main, Germany. ${ }^{3}$ Klinikum Kempten, Klinikverbund Allgäu, Kempten/Allgäu, Germany. ${ }^{4}$ Department of Pediatrics, Helios Dr. Horst Schmidt Clinic Wiesbaden, Wiesbaden, Germany. ${ }^{5}$ Department of Pediatrics, University Medicine Mainz, Mainz, Germany. ${ }^{6}$ St. Bonifatius Hospital, Lingen, Germany. ${ }^{7}$ Epilepsy Center Kleinwachau, Radeberg, Germany. ${ }^{8}$ Department of Neuropediatrics, Vivantes Klinikum Neukölln, Berlin, Germany. ${ }^{9}$ Department of Neuropediatrics, University Hospital for Children and Adolescents, Rostock, Germany. ${ }^{10}$ Epilepsy Center Hessen and Department of Neurology, Philipps-University Marburg, Marburg (Lahn), Germany. ${ }^{11}$ Departments of Clinical Neurosciences, Medical Genetics, and Community Health Sciences, Hotchkiss Brain Institute and Alberta Children's Hospital Research Institute, Cumming School of Medicine, University of Calgary, Calgary, AB, Canada.

${ }^{12}$ Department of Pediatric Neurology, Psychosomatics and Pain Management, Klinikum Stuttgart, Stuttgart, Germany. ${ }^{13}$ Department of Neuropediatrics, Children's Hospital at University Medical Center Homburg, Homburg, Germany. ${ }^{14}$ Department of Neurology, Epilepsy Center, University Medicine Greifswald, Greifswald, Germany. ${ }^{15}$ Division of Nephrology, Medizinische Klinik und Poliklinik IV, Klinikum der LMU München - Innenstadt, München, Germany. ${ }^{16}$ Department of Nephrology, Klinikum Rechts Der Isar, Technische Universität München, München, Germany. ${ }^{17}$ Department of Neuropediatrics and Socialpediatrics, University Hospital of Ruhr University Bochum, Bochum, Germany. ${ }^{18}$ Department of Nephrology and Internal Intensive Care, Charité - University Medicine Berlin, Berlin, Germany. ${ }^{19}$ Department of Neuropediatrics, GoetheUniversity Frankfurt, Frankfurt am Main, Germany.

Received: 19 December 2020 Accepted: 22 April 2021

Published online: 02 June 2021

\section{References}

1. Ebrahimi-Fakhari D, Mann LL, Poryo M, Graf N, von Kries R, Heinrich B, Ebrahimi-Fakhari D, Flotats-Bastardas M, Gortner L, Zemlin M, et al. Incidence of tuberous sclerosis and age at first diagnosis: new data and emerging trends from a national, prospective surveillance study. Orphanet J Rare Dis. 2018;13(1):117.

2. Hong $\mathrm{CH}$, Darling TN, Lee $\mathrm{CH}$. Prevalence of tuberous sclerosis complex in Taiwan: a national population-based study. Neuroepidemiology. 2009;33(4):335-41.

3. Shepherd CW, Beard CM, Gomez MR, Kurland LT, Whisnant JP. Tuberous sclerosis complex in Olmsted County, Minnesota, 1950-1989. Arch Neurol. 1991;48(4):400-1.

4. Zöllner JP, Franz DN, Hertzberg C, Nabbout R, Rosenow F, Sauter M, Schubert-Bast S, Wiemer-Kruel A, Strzelczyk A. A systematic review on the burden of illness in individuals with tuberous sclerosis complex (TSC). Orphanet J Rare Dis. 2020;15(1):23. 
5. O'Callaghan FJ, Shiell AW, Osborne JP, Martyn CN. Prevalence of tuberous sclerosis estimated by capture-recapture analysis. Lancet. 1998;351(9114):1490.

6. Hong CH, Tu HP, Lin JR, Lee $\mathrm{CH}$. An estimation of the incidence of tuberous sclerosis complex in a nationwide retrospective cohort study (1997-2010). Br J Dermatol. 2016;174(6):1282-9.

7. Curatolo P, Moavero R, de Vries PJ. Neurological and neuropsychiatric aspects of tuberous sclerosis complex. Lancet Neurol. 2015;14(7):733-45.

8. Chu-Shore CJ, Major P, Camposano S, Muzykewicz D, Thiele EA. The natural history of epilepsy in tuberous sclerosis complex. Epilepsia. 2010;51(7):1236-41.

9. Skalicky AM, Rentz AM, Liu Z, Wheless JW, Pelletier CL, Dunn DW, Frost MD, Nakagawa J, Magestro M, Prestifilippo J, et al. The burden of subependymal giant cell astrocytomas associated with tuberous sclerosis complex: results of a patient and caregiver survey. J Child Neurol. 2015;30(5):563-9.

10. Kingswood JC, Belousova E, Benedik MP, Carter T, Cottin V, Curatolo P, Dahlin M, D'Amato L, Beaure d'Augeres G, de Vries PJ, et al. Renal manifestations of tuberous sclerosis complex: key findings from the final analysis of the TOSCA study focussing mainly on renal angiomyolipomas. Front Neurol. 2020;11:972.

11. Kristof AS, Zhi Li P, Major P, Landry JS. Lymphangioleiomyomatosis and tuberous sclerosis complex in Quebec: prevalence and health-care utilization. Chest. 2015;148(2):444-9.

12. Sancak O, Nellist M, Goedbloed M, Elfferich P, Wouters C, Maat-Kievit A, Zonnenberg B, Verhoef S, Halley D, van den Ouweland A. Mutational analysis of the TSC1 and TSC2 genes in a diagnostic setting: genotypephenotype correlations and comparison of diagnostic DNA techniques in Tuberous Sclerosis Complex. Eur J Hum Genet. 2005;13(6):731-41.

13. Rosset C, Netto CBO, Ashton-Prolla P. TSC1 and TSC2 gene mutations and their implications for treatment in Tuberous Sclerosis Complex: a review. Genet Mol Biol. 2017;40(1):69-79.

14. Schubert-Bast S, Rosenow F, Klein KM, Reif PS, Kieslich M, Strzelczyk A. The role of mTOR inhibitors in preventing epileptogenesis in patients with TSC: current evidence and future perspectives. Epilepsy Behav. 2019;91:94-8.

15. Kingswood JC, Crawford P, Johnson SR, Sampson JR, Shepherd C, Demuth D, Erhard C, Nasuti P, Patel K, Myland M, et al. The economic burden of tuberous sclerosis complex in the UK: a retrospective cohort study in the Clinical Practice Research Datalink. J Med Econ. 2016;19(11):1087-98.

16. Shepherd C, Koepp M, Myland M, Patel K, Miglio C, Siva V, Gray E, Neary M. Understanding the health economic burden of patients with tuberous sclerosis complex (TSC) with epilepsy: a retrospective cohort study in the UK Clinical Practice Research Datalink (CPRD). BMJ Open. 2017;7(10):e015236.

17. Jansen AC, Vanclooster S, de Vries PJ, Fladrowski C, Beaure d'Augeres G, Carter T, Belousova E, Benedik MP, Cottin V, Curatolo P, et al. Burden of illness and quality of life in tuberous sclerosis complex: findings from the TOSCA Study. Front Neurol. 2020;11:904.

18. Henske EP, Jozwiak S, Kingswood JC, Sampson JR, Thiele EA. Tuberous sclerosis complex. Nat Rev Dis Primers. 2016;2:16035.

19. Northrup $H$, Krueger DA. International tuberous sclerosis complex consensus G: tuberous sclerosis complex diagnostic criteria update: recommendations of the 2012 international tuberous sclerosis complex consensus conference. Pediatr Neurol. 2013:49(4):243-54.

20. Fisher RS, Cross JH, French JA, Higurashi N, Hirsch E, Jansen FE, Lagae L, Moshe SL, Peltola J, Roulet Perez E, et al. Operational classification of seizure types by the international league against epilepsy: position paper of the ILAE commission for classification and terminology. Epilepsia. 2017:58(4):522-30.

21. Scheffer IE, Berkovic S, Capovilla G, Connolly MB, French J, Guilhoto L, Hirsch E, Jain S, Mathern GW, Moshe SL, et al. ILAE classification of the epilepsies: position paper of the ILAE Commission for Classification and Terminology. Epilepsia. 2017;58(4):512-21.

22. von Elm E, Altman DG, Egger M, Pocock SJ, Gotzsche PC, Vandenbroucke JP, Initiative S. The Strengthening the Reporting of Observational Studies in Epidemiology (STROBE) statement: guidelines for reporting observational studies. PLoS Med. 2007;4(10):e296.

23. Riechmann J, Strzelczyk A, Reese JP, Boor R, Stephani U, Langner C, Neubauer BA, Oberman B, Philippi H, Rochel M, et al. Costs of epilepsy and cost-driving factors in children, adolescents, and their caregivers in Germany. Epilepsia. 2015;56(9):1388-97.

24. Strzelczyk A, Nickolay T, Bauer S, Haag A, Knake S, Oertel WH, Reif PS, Rosenow F, Reese JP, Dodel R, et al. Evaluation of health-care utilization among adult patients with epilepsy in Germany. Epilepsy Behav. 2012;23(4):451-7.

25. Strzelczyk A, Schubert-Bast S, Bast T, Bettendorf U, Fiedler B, Hamer HM, Herting A, Kalski M, Kay L, Kieslich M, et al. A multicenter, matched case-control analysis comparing burden-of-illness in Dravet syndrome to refractory epilepsy and seizure remission in patients and caregivers in Germany. Epilepsia. 2019;60(8):1697-710.

26. Willems LM, Richter S, Watermann N, Bauer S, Klein KM, Reese JP, Schoffski O, Hamer HM, Knake S, Rosenow F, et al. Trends in resource utilization and prescription of anticonvulsants for patients with active epilepsy in Germany from 2003 to 2013-a ten-year overview. Epilepsy Behav. 2018;83:28-35.

27. Graf von der Schulenburg JM, Greiner W, Jost F, Klusen N, Kubin M, Leidl R, Mittendorf T, Rebscher H, Schoeffski O, Vauth C, et al. German recommendations on health economic evaluation: third and updated version of the Hanover Consensus. Value Health. 2008;11(4):539-44.

28. Schwabe U, Paffrath D, Ludwig W-D, Klauber J. ArzneiverordnungsReport. Berlin: Springer; 2019.

29. Bock JO, Brettschneider C, Seidl H, Bowles D, Holle R, Greiner W, Konig $\mathrm{HH}$. Calculation of standardised unit costs from a societal perspective for health economic evaluation. Gesundheitswesen (Bundesverband der Arzte des Offentlichen Gesundheitsdienstes (Germany)). 2015;77(1):53-61.

30. Bayat A, Hjalgrim H, Moller RS. The incidence of SCN1A-related Dravet syndrome in Denmark is 1:22,000: a population-based study from 2004 to 2009. Epilepsia. 2015;56(4):e36-39.

31. Dravet $\mathrm{C}$, Oguni H. Dravet syndrome (severe myoclonic epilepsy in infancy). Handb Clin Neurol. 2013;111:627-33.

32. Forsgren L, Nystrom L. An incident case-referent study of epileptic seizures in adults. Epilepsy Res. 1990;6(1):66-81.

33. Barber JA, Thompson SG. Analysis of cost data in randomized trials: an application of the non-parametric bootstrap. Stat Med. 2000;19(23):3219-36.

34. Desgagne A, Castilloux AM, Angers JF, LeLorier J. The use of the bootstrap statistical method for the pharmacoeconomic cost analysis of skewed data. Pharmacoeconomics. 1998;13(5 Pt 1):487-97.

35. Friede T, Posch M, Zohar S, Alberti C, Benda N, Comets E, Day S, Dmitrienko A, Graf A, Gunhan BK, et al. Recent advances in methodology for clinical trials in small populations: the InSPiRe project. Orphanet J Rare Dis. 2018;13(1):186.

36. Gaasterland CMW, van der Weide MCJ, du Prie-Olthof MJ, Donk M, Kaatee MM, Kaczmarek R, Lavery C, Leeson-Beevers K, O'Neill N, Timmis $\mathrm{O}$, et al. The patient's view on rare disease trial design-A qualitative study. Orphanet J Rare Dis. 2019;14(1):31

37. Song X, Liu Z, Cappell K, Gregory C, Said Q, Prestifilippo J, Charles H, Hulbert J, Bissler J. Healthcare utilization and costs in patients with tuberous sclerosis complex-related renal angiomyolipoma. J Med Econ. 2017;20(4):388-94.

38. Sun P, Liu Z, Krueger D, Kohrman M. Direct medical costs for patients with tuberous sclerosis complex and surgical resection of subependymal giant cell astrocytoma: A US national cohort study. J Med Econ. 2015;18(5):349-56.

39. Strzelczyk A, Reese JP, Dodel R, Hamer HM. Cost of epilepsy: A systematic review. Pharmacoeconomics. 2008;26(6):463-76.

40. Vekeman F, Magestro M, Karner P, Duh MS, Nichols T, van Waalwijk van Doorn-Khosrovani SB, Zonnenberg BA. Kidney involvement in tuberous sclerosis complex: The impact on healthcare resource use and costs. J Med Econ. 2015;18(12):1060-70.

41. Kingswood JC, Nasuti P, Patel K, Myland M, Siva V, Gray E. The economic burden of tuberous sclerosis complex in UK patients with renal manifestations: A retrospective cohort study in the clinical practice research datalink (CPRD). J Med Econ. 2016;19(12):1116-26.

42. Wilson TA, Rodgers S, Tanweer O, Agarwal P, Lieber BA, Agarwal N, McDowell M, Devinsky O, Weiner H, Harter DH. Tuberous sclerosis health care utilization based on the national inpatient sample database: A review of 5655 hospitalizations. World Neurosurg. 2016:91:97-105. 
43. Skalicky AM, Rentz AM, Liu Z, Said Q, Nakagawa JA, Frost MD, Wheless JW, Dunn DW. Economic burden, work, and school productivity in individuals with tuberous sclerosis and their families. J Med Econ. 2018;21(10):953-9.

44. Betts KA, Stockl KM, Yin L, Hollenack K, Wang MJ, Yang X. Economic burden associated with tuberous sclerosis complex in patients with epilepsy. Epilepsy Behav. 2020;112:107494.

45. Chu WC, Chiang LL, Chan DC, Wong WH, Chan GC. Prevalence, mortality and healthcare economic burden of tuberous sclerosis in Hong Kong: A population-based retrospective cohort study (1995-2018). Orphanet J Rare Dis. 2020;15(1):264

46. European Medicines Agency: Votubia—Summary of Opinion (Post Authorisation) In. London, UK: European Medicines Agency; 2012

47. Strzelczyk A, Grau J, Bast T, Bertsche A, Bettendorf U, Hahn A, Hartmann $H$, Hertzberg C, Hornemann F, Immisch I, et al. Prescription patterns of antiseizure drugs in Tuberous sclerosis complex (TSC)-associated epilepsy: a multicenter cohort study from Germany and review of the literature. Expert Rev Clin Pharmacol. 2021. https://doi.org/10.1080/17512 433.17512021.11911643.

48. Strzelczyk A, Griebel C, Lux W, Rosenow F, Reese JP. The Burden of severely drug-refractory epilepsy: A comparative longitudinal evaluation of mortality, morbidity, resource use, and cost using German health insurance data. Front Neurol. 2017;8:712.

49. Strzelczyk A, Kalski M, Bast T, Wiemer-Kruel A, Bettendorf U, Kay L, Kieslich M, Kluger G, Kurlemann G, Mayer T, et al. Burden-of-illness and cost-driving factors in Dravet syndrome patients and carers: A prospective, multicenter study from Germany. Eur J Paediatr Neurol. 2019;23(3):392-403.

50. Strzelczyk A, Schubert-Bast S, Simon A, Wyatt G, Holland R, Rosenow F. Epidemiology, healthcare resource use, and mortality in patients with probable Lennox-Gastaut syndrome: A population-based study on German health insurance data. Epilepsy Behav. 2021:115:107647.

51. Kohlhase K, Zöllner JP, Tandon N, Strzelczyk A, Rosenow F. Comparison of minimally invasive and traditional surgical approaches for refractory mesial temporal lobe epilepsy: A systematic review and meta-analysis of outcomes. Epilepsia. 2021;62 (4):831-845.

52. Thiele EA, Bebin EM, Bhathal H, Jansen FE, Kotulska K, Lawson JA, O'Callaghan FJ, Wong M, Sahebkar F, Checketts D. et al. Add-On cannabidiol treatment for drug-resistant seizures in tuberous sclerosis complex: A placebo-controlled randomized clinical trial. JAMA Neurol. 2021:78(3):1-9.

53. Józwiak J, Sontowska I, Ploski R. Frequency of TSC 1 and TSC2 mutations in American, British, Polish and Taiwanese populations. Mol Med Rep. 2013;8(3):909-13.

54. Klug C, Schreiber-Katz O, Thiele S, Schorling E, Zowe J, Reilich P, Walter MC, Nagels KH. Disease burden of spinal muscular atrophy in Germany. Orphanet J Rare Dis. 2016;11(1):58.

55. Schreiber-Katz O, Klug C, Thiele S, Schorling E, Zowe J, Reilich P, Nagels $\mathrm{KH}$, Walter MC. Comparative cost of illness analysis and assessment of health care burden of Duchenne and Becker muscular dystrophies in Germany. Orphanet J Rare Dis. 2014;9:210.

56. Willems LM, Hamer HM, Knake S, Rosenow F, Reese JP, Strzelczyk A. General trends in prices and prescription patterns of anticonvulsants in Germany between 2000 and 2017: Analysis of national and Cohort-based data. Appl Health Econ Health Policy. 2019;17(5):707-22.

57. Begley CE, Annegers JF, Lairson DR, Reynolds TF. Methodological issues in estimating the cost of epilepsy. Epilepsy Res. 1999;33(1):39-55.

58. Koopmanschap MA, Rutten FF, van Ineveld BM, van Roijen L. The friction cost method for measuring indirect costs of disease. J Health Econ. 1995;14(2):171-89.

59. Institute for Quality and Efficiency in Health Care: (IQWiG) - Technical Document on Cost Estimation 09.10.2008.

60. Schöffski O, v.d. Schulenburg J: Gesundheitsökonomische Evaluationen, 3. vollständig überarbeitete Auflage, Berlin: Springer; 2007.

61. v.d. Schulenburg JM, Greiner W, Jost F, Klusen N, Kubin M, Leidl R, Mittendorf T, Rebscher H, Schoeffski O, Vauth C, et al. German recommendations on health economic evaluation: third and updated version of the Hanover Consensus. Value Health 2008;11(4):539-544.

\section{Publisher's Note}

Springer Nature remains neutral with regard to jurisdictional claims in published maps and institutional affiliations.
Ready to submit your research? Choose BMC and benefit from:

- fast, convenient online submission

- thorough peer review by experienced researchers in your field

- rapid publication on acceptance

- support for research data, including large and complex data types

- gold Open Access which fosters wider collaboration and increased citations

- maximum visibility for your research: over $100 \mathrm{M}$ website views per year

At BMC, research is always in progress.

Learn more biomedcentral.com/submissions 